\title{
The Effects of Moderate Neuromuscular Blockade Combined with Transverse Abdominal Plane Block on Surgical Space Conditions During Laparoscopic Colorectal Surgery: A Randomised Clinical Study
}

\section{Fang Ke}

Ruijin Hospital, Shanghai Jiaotong University School of Medicine

\section{Zijin Shen}

Ruijin Hospital, Shanghai Jiaotong University School of Medicine

\section{Cheng Wu}

Naval Medical University

\section{Lin Zhang}

Ruijin Hospital, Shanghai Jiaotong University School of Medicine

Rong Dong ( $\sim$ sally9132@163.com )

Ruijin Hospital, Shanghai Jiaotong University School of Medicine

\section{Research Article}

Keywords: transverse abdominal plane block (TAPB), neuromuscular blockade monitoring, surgical space conditions

Posted Date: September 27th, 2021

DOI: https://doi.org/10.21203/rs.3.rs-820460/v1

License: (c) (i) This work is licensed under a Creative Commons Attribution 4.0 International License.

Read Full License

Version of Record: A version of this preprint was published at BMC Anesthesiology on April 4th, 2022. See the published version at https://doi.org/10.1186/s12871-022-01623-7. 


\section{Abstract \\ Background}

Deep neuromuscular blockade may be beneficial on surgical space conditions during laparoscopic surgery. The effects of moderate neuromuscular blockade combined with transverse abdominal plane block (TAPB) on the surgical space conditions during laparoscopic surgery has not been described. We investigated if moderate neuromuscular blockade combined with TAPB would be associated with similar surgical space conditions compared with deep neuromuscular blockade.

\section{Methods}

Eighty patients undergoing elective laparoscopic surgery for colorectal cancer were randomly divided into two groups. The intervention group was treated with moderate neuromuscular blockade (train-of-four (TOF) count between 1 and 3) combined with TAPB (M group), while the control group was treated with deep neuromuscular blockade (D group), with a TOF count of 0 and a post-tetanic count (PTC) $\geq 1$. Both groups received the same anesthesia management. The distance between the sacral promontory and the umbilical skin during the operation was compared between the two groups. The surgeon scored the surgical space conditions according to a five-point ordinal scale. Patients' pain scores were evaluated eight hours after the operation.

\section{Results}

The $95 \%$ confidence intervals of the difference in the distance from the sacral promontory to the umbilical skin between the groups were $-1.45-0.77 \mathrm{~cm}$. According to the preset non-inferior standard of $1.5 \mathrm{~cm},(-1.45, \infty)$ completely fell within $(-1.50, \infty)$, and the non-inferior effect test was qualified. There was no significant difference in the surgical rating score between the two groups. The dosage of rocuronium in group $\mathrm{D}$ was significantly higher than that in group $\mathrm{M}(P<0.01)$. The $\mathrm{M}$ group had significantly lower pain scores than the $\mathrm{D}$ group eight hours after the operation $(P<0.05)$.

\section{Conclusions}

In laparoscopic colorectal cancer surgery, moderate neuromuscular blockade combined with TAPB can provide surgical space conditions similar to those of deep neuromuscular blockade, and at the same time, reduces the use of muscle relaxants, relieves postoperative pain within 4 hours after operation, shorten the time to extubation and stay in PACU.

\section{Trial registration:}


chictr.org.cn (ChiCTR2000034621), registered on 12, July, 2020

\section{Background}

During earlier years of laparoscopic surgery development, most anesthesiologists usually adopted a moderate neuromuscular blockade due to the minimally invasive incision.[1, 2] However, pneumoperitoneum can develop when using a moderate neuromuscular blockade, leading to reduced lung compliance, decreased functional residual capacity, and atelectasis. In terms of long-term pneumoperitoneum, carbon dioxide accumulation can cause hypercapnia and acidosis, resulting in insufficient perfusion of the abdominal organs and hemodynamic fluctuations.[3, 4]

In recent years, anesthesiologists have applied deep neuromuscular blockade in laparoscopic surgery to improve surgical space conditions.[5-7] So as to reduce the postoperative pain and shorten the recovery time.[8-10] However, deep neuromuscular blockade may increase the risk of residual neuromuscular blockade after the operation, leading to respiratory complications.[11-13]

Local anesthetics could affect neuromuscular junction conduction through complex presynaptic and postsynaptic effects, thus enhancing the effect of non depolarizing muscle relaxants.[14, 15] To the authors' knowledge, no published studies have investigated the impact of moderate neuromuscular blockade with transverse abdominal plane block (TAPB) on surgical space conditions during laparoscopic surgery. During our pilot study, moderate neuromuscular blockade with TAPB could provide better surgical space conditions than moderate neuromuscular blockade alone.

We designed this study to assess the the effects of moderate neuromuscular blockade with TAPB to deep neuromuscular blockade on surgical space conditions during laparoscopic surgery for colorectal cancer. We hypothesized that moderate neuromuscular blockade with TAPB was non-inferior to deep blockade in providing "optimal" surgical space conditions as assessed by the surgeon.

\section{Methods}

This trail was approved by the ethics committee of the Ruijin Hospital affiliated to the Shanghai Jiaotong University School of Medicine (No. 2020-007-1) and written informed consent was obtained from all subjects participating in the trial. The trial was registered prior to patient enrollment at chictr.org.cn (ChiCTR2000034621, Principal investigator: Fang Ke, Date of registration: 12, July, 2020).

In this study, patients undergoing surgery for colorectal cancer were enrolled on an elective date from August 2020 to February 2021. Patients had an American Society of Anesthesiologists (ASA) grade I-III physical status classification, were between 18-80 years of age, and had a body mass index (BMI) between $18-26 \mathrm{~kg} / \mathrm{m}^{2}$. Patients suffering from neuromuscular junction diseases, severe heart, lung, liver, or kidney insufficiency, severe blood system disease, coagulation dysfunction, thrombocytopenia, or hemophilia were excluded from the study. 
Participants were randomly divided into two groups: a deep neuromuscular blockade group (D Group) or a moderate neuromuscular blockade combined with TAPB group (M Group). A random number table method was adopted using Excel to allocate participants to the groups, and the grouping results were only known by the anesthesiologist. Throughout the operation, the patient's blood pressure, electrocardiogram, pulse oxygen saturation, and Narcotrend (Monitor Technik, Bad Bramstedt, Germany) were routinely monitored. Oxygen was inhaled through a mask, and peripheral veins were catheterized for fluid administration.

A neuromuscular blockade monitor (E-NMT, GE Company, Finland) was used to stimulate the ulnar nerve and observe the contraction reaction of the adductor pollicis muscle. TOF counting with a frequency of $2 \mathrm{~Hz}$, current of $60 \mathrm{~mA}$, at 20 -s intervals was adopted. Every $10 \mathrm{~min}$, PTC was monitored in the D Group.

Propofol $(2 \mathrm{mg} / \mathrm{kg})$ and sufentanil $(0.3 \mu \mathrm{g} / \mathrm{kg})$ were given for anesthesia induction. After the patient lost consciousness, TOF count monitoring was performed, ensuring the basic values were $90-110 \%$ for three consecutive times. The D Group was given rocuronium $\left(0.6 \mathrm{mg} / \mathrm{kg}\right.$, Esmeron ${ }^{\circledR}$, N.V. Organon, Netherlands) through intravenous injection, while the $M$ Group was given rocuronium $(0.4 \mathrm{mg} / \mathrm{kg})$ intravenously. Continuous monitoring of neuromuscular blockade was conducted, and endotracheal intubation was performed when the TOF count was 0 .

After endotracheal intubation, the researchers implemented TAPB according to the patient's group. The M Group received ultrasound-guided bilateral TAPB using ultrasound (Edge, Sonosite ${ }^{\circledR}$, Fuji Film, Japan) and in-plane techniques. The patients lay supine, exposing the abdominal area between the costal margin and iliac crest. The ultrasound probe was placed transversely between the costal margin and the iliac crest, near the front or middle axillary line. Three layers of abdominal muscles were identified, including the external oblique muscle, the internal oblique muscle, and the transverse abdominal muscle. After the needle (Stimuplex ${ }^{\circledR}$ D, $0.71 * 120 \mathrm{~mm} 22 \mathrm{G} *$, B Braun Melsungen AG, Germany) was inserted between the internal oblique and transverse abdominal muscles, it was withdrawn to ensure that the needle tip was not in a blood vessel. Then local anesthetic $\left(20 \mathrm{ml}\right.$ of $0.375 \%$ ropivacaine, Naropin ${ }^{\circledR}$, AstraZeneca) was injected into each side.

Within 10 min of TAPB administration, the surgeon entered the room and prepped and draped the patient. Pneumoperitoneum was established five minutes after the operation commenced. Then, the surgeon (observer) measured the distance from the sacral promontory to the umbilical skin, while pneumoperitoneum pressure was controlled at $12 \mathrm{mmHg} .[5,16]$ During the operation, the surgeon scored the surgical space conditions according to a five-point ordinal scale ranging from 1 (extremely poor conditions) to 5 (optimal conditions) (Table 1). [17] The data were recorded by the anesthesiologist. The surgeon didn't know the group allocation. 
Table 1

The surgical rating score

1 Extremely poor conditions: the surgeon is unable to work because of coughing or because of the inability to obtain a visible laparoscopic field because of inadequate muscle relaxation. Additional neuromuscular blocking agents must be given

2 Poor conditions: there is a visible laparoscopic field, but the surgeon is severely hampered by inadequate muscle relaxation with continuous muscle contractions, movements, or both with the hazard of tissue damage. Additional neuromuscular blocking agents must be given

3 Acceptable conditions: there is a wide visible laparoscopic field but muscle contractions, movements, or both occur regularly causing some interference with the surgeon's work. There is the need for additional neuromuscular blocking agents to prevent deterioration

4 Good conditions: there is a wide laparoscopic working field with sporadic muscle contractions, movements, or both. There is no immediate need for additional neuromuscular blocking agents unless there is the fear of deterioration

5 Optimal conditions: there is a wide visible laparoscopic working field without any movement or contractions. There is no need for additional neuromuscular blocking agents

Anesthesia was maintained by target control infusion (TCl) and desflurane. The TCl(Perfusor ${ }^{\circledR}$ Space Infusion Pump, B.Braun Melsungen AG.) effect compartment concentration(Marsh model) of propofol was $1 \mu \mathrm{g} / \mathrm{ml}$, and remifentanil was sustainedly infused at $0.1 \mu \mathrm{g} / \mathrm{kg} / \mathrm{min}$. Desflurane (6\%) was inhaled continuously, and the minimum alveolar concentration (MAC) was maintained between 0.7 and 1.0. The depth of anesthesia was maintained in the Narcotrend range of 30-50. A single dose of sufentanil $(0.2 \mu \mathrm{g} / \mathrm{kg})$ was given before incision. Neuromuscular blockade was monitored and maintained by continuously infusing rocuronium (initial rate of $3 \mu \mathrm{g} / \mathrm{kg} / \mathrm{min}$ ). The TOF count in the D Group was maintained at 0 with PTC $\geq 1$. [18] The PTC was measured every 10 min during the operation. When PTC was $>15$, the infusing rate of rocuronium was increased, and when PTC was $<1$, infusing rate was decreased. If TOF count occurred during the period, a single dose of rocuronium (5mg) was administered. The TOF count in the M Group was maintained within 1-3.[18] The rocuronium infusing rate was increased when TOF count was 3 and decreased when TOF count was 1 (Fig. 1). When systolic blood pressure decreased by $>30 \%$ or mean arterial pressure (MAP) $<65 \mathrm{mmHg}$ for more than three minutes, phenylephrine $(20 \mu \mathrm{g})$ or ephedrine $(5 \mathrm{mg})$ was injected intravenously once.

The infusion of muscle relaxants ceased and a single dose of sufentanil $(0.3 \mu \mathrm{g} / \mathrm{kg})$ were given $10 \mathrm{~min}$ before the end of the operation. Then the patients were transferred to the post-anesthesia care unit as the standard procedure at author's institution. In this study, all patients were routinely antagonized. When the TOF count recovered to 0.1 , neostigmine $(40 \mu \mathrm{g} / \mathrm{kg}$, Xinyi, China) and atropine $(20 \mu \mathrm{g} / \mathrm{kg}$, Xinyi, China) were given to antagonize the residual effects of the muscle relaxants. Coughing and swallowing reflexes were recovered when the patient regained consciousness. The endotracheal tube was removed after TOF ratio $₫ 0.9$, establishing a steady breathing frequency of $10-20$ breathes per minute and $\mathrm{PetCO}_{2}$ was $\leq$ $45 \mathrm{mmHg}$. Time to extubation, total time in PACU and other adverse events (apnea, desaturation) were recorded. 
Oxycodone was used for patient-controlled-analgesia (PCA), the background dose was $1 \mathrm{mg} / \mathrm{h}$, bolus dose was $1 \mathrm{mg} /$ time, locked for 30 minutes. The postoperative visual analogue scale (VAS) and bolus time follow-up were performed by a specialized anesthetic nurse. The specialized anesthetic nurse didn't know the group allocation.

\section{Outcome Measures}

The primary outcome measure was the distance from the sacral promontory to the umbilical skin and the surgeon's subjective score of the surgical space conditions. Secondary outcome measures included the dosage of various narcotic drugs, the patient's hemodynamic parameters during different periods of the operation, and patient pain scores (VAS) after the operation.

\section{Statistical analysis}

The purpose of this clinical trial was to verify that the surgical space conditions of the M Group were not inferior to that of the $D$ Group. Therefore, the sample size estimation formula for a non-inferior, parallel (1:1) clinical trial was: $N_{c}=\left(Z_{1-a}+Z_{1-\beta}\right) \sigma^{2}(1+1 / K) /\left(\mu_{T}-\mu_{C}+\Delta\right)^{2}$. According to the results of our pilot study, the mean $\mu_{\mathrm{T}}$ value of the distance from the sacral promontory to the umbilical skin in the M Group was $16.03 \mathrm{~cm}$, and the mean $\mu_{\mathrm{C}}$ value in the $\mathrm{D}$ Group was $15.66 \mathrm{~cm}$. The non-inferiority limit was set as $\Delta=1.5$ $\mathrm{cm}$. Assuming that the standard deviation $(\sigma)$ was the same for both groups at 2.4 , and $a=0.025, \beta=$ 0.10 , and $K=1$, then according to the formula, $n_{c}=35$. Considering a drop-out rate of $10 \%$ in each group, the number of cases in each group should be no less than 39 . Thus, 40 cases were included in each of the two groups in this study, which fulfills the requirements of the statistical tests.

Measurement data were summarized as mean \pm standard deviation $(\nabla x \pm s)$. Categorical data were summarized as numbers and percentages(\%). Comparisons between the two groups were analyzed by student t-test for measurement data, Chi-square test was used for categorical data (or Fisher's exact test if expected count less than five), and Wilcoxon rank-sum test was used for ranked data. Bilateral $95 \%$ confidence intervals were calculated of the difference in surgical space measurements between the groups. Whether the effect of moderate neuromuscular blockade combined with TAPB on surgical space measurements was not inferior to that of deep neuromuscular blockade was judged according to the preset non-inferior effect limit of $1.5 \mathrm{~cm}$. If the lower boundary of the $95 \%$ confidence interval for $\left(\mu_{\mathrm{T}}-\mu_{\mathrm{C}}\right)$ did not cross $-1.5 \mathrm{~cm}$, noninferiority of the $M$ group to the $D$ group would be established. A $P$ value of < 0.05 was considered statistically significant. Statistical analysis was performed with SPSS20.0 software.

\section{Results}

Among the 155 patients screened for inclusion in the study, 73 refused to sign informed consent and 82 participated in the study, of which 2 patients were excluded due to signal disturbance during neuromuscular blockade monitoring. 80 patients were finally included in the statistical analysis. Patients 
were randomly divided into the $D(n=40)$ and $M(n=40)$ Groups according to the different neuromuscular blockade management schemes(Fig. 2). There were no significant differences in sex, age, body mass, surgical category, and preoperative examination between the two groups $(P<0.05$, Table 2$)$.

Table 2

Baseline demographic and clinical characteristics for each group

\begin{tabular}{|llll|}
\hline & $\begin{array}{l}\text { D Group } \\
(\mathbf{n}=\mathbf{4 0})\end{array}$ & $\begin{array}{l}\text { M Group } \\
(\mathbf{n}=\mathbf{4 0})\end{array}$ & \\
\hline ASA(I/II/III) & $8 / 28 / 4$ & $11 / 27 / 2$ & 0.309 \\
\hline Gender (male/female) & $24 / 16$ & $24 / 16$ & 1.000 \\
\hline Age (years) & $60 \pm 1$ & $60 \pm 2$ & 0.972 \\
\hline Height (cm) & $163 \pm 1.3$ & $166 \pm 1.1$ & 0.049 \\
\hline Weight (kg) & $63 \pm 1.5$ & $64 \pm 1.5$ & 0.490 \\
\hline BMI (kg/m $\left.{ }^{2}\right)$ & $23.6 \pm 0.47$ & $23.4 \pm 0.42$ & 0.728 \\
\hline Total bilirubin (umol/L) & $11.73 \pm 0.68$ & $13.75 \pm 0.74$ & 0.049 \\
\hline Direct bilirubin (umol/L) & $2.28 \pm 0.14$ & $2.19 \pm 0.13$ & 0.665 \\
\hline Total protein (g/L) & $67.85 \pm 0.84$ & $67.68 \pm 0.74$ & 0.876 \\
\hline Albumin (g/L) & $39.2 \pm 0.5$ & $39 \pm 0.5$ & 0.786 \\
\hline Creatinine (umol/L) & $78.1 \pm 2.2$ & $79.8 \pm 2.3$ & 0.586 \\
\hline Urea nitrogen (mmol/L) & $5.23 \pm 0.23$ & $4.88 \pm 0.2$ & 0.263 \\
\hline Hemoglobin (g/L) & $123 \pm 3.4$ & $129 \pm 2.7$ & 0.199 \\
\hline Hematocrit & $0.43 \pm 0.03$ & $0.37 \pm 0.01$ & 0.390 \\
\hline ASA = American Society of Anesthesiologists; BMI= body mass index. \\
\hline
\end{tabular}

The distance from the sacral promontory to the umbilical skin after pneumoperitoneum was $16.03 \pm$ $2.17 \mathrm{~cm}$ in $\mathrm{D}$ group and $16.37 \pm 2.78 \mathrm{~cm}$ in $\mathrm{M}$ group. The $95 \%$ confidence interval of the difference in the distance between the $M$ and $D$ groups was $-1.45 \sim 0.77 \mathrm{~cm}$. The lower boundary of this $95 \% \mathrm{Cl}$ did not cross $-1.5 \mathrm{~cm}$, and as such, the noninferiority of the $M$ group to the $D$ group was established.

There was no difference in the surgical rating score of the surgical space conditions after the operation between the two groups. In the D Group, there were 34 patients with a score of 5 points, five patients with a score of 4 points, and one patient with a score of 3 points. In the $\mathrm{M}$ group, 35 patients scored 5 points, and five patients had a score of 4 points. 
With the exception of the different schemes for neuromuscular blockade management, other drugs for maintaining anesthesia were the same in both groups. The rocuronium dosage in the $D$ Group was significantly higher than that of the M Group $(83.6 \pm 3.6 \mathrm{mg} v s 69.2 \pm 3.1 \mathrm{mg}, P<0.01)$. There were no significant differences in the fluid balance, the duration of anesthesia, operative time, and pneumoperitoneum time between the two groups $(P>0.05$, Table 3$)$. 
Table 3

Comparison of primary and secondary outcomes between the two groups

\begin{tabular}{|c|c|c|c|}
\hline & $\begin{array}{l}\text { D Group } \\
(n=40)\end{array}$ & $\begin{array}{l}\text { M Group } \\
(n=40)\end{array}$ & $P$ \\
\hline \multicolumn{4}{|l|}{ Primary outcome measures } \\
\hline Surgical space measurement $(\mathrm{cm})$ & $16.03 \pm 2.17$ & $16.37 \pm 2.78$ & \\
\hline Surgical rating score ( $1 / 2 / 3 / 4 / 5$ points) & $0 / 0 / 1 / 5 / 34$ & $0 / 0 / 0 / 5 / 35$ & 0.717 \\
\hline \multicolumn{4}{|l|}{ Secondary outcome measures } \\
\hline Rocuronium bromide (mg) & $83.6 \pm 3.6$ & $69.2 \pm 3.1$ & 0.003 \\
\hline Remifentanil (mg) & $0.99 \pm 0.08$ & $1.01 \pm 0.08$ & 0.435 \\
\hline Propofol (mg) & $510 \pm 35.7$ & $524 \pm 32.8$ & 0.770 \\
\hline Sufentanil $(\mu g)$ & $46 \pm 1.2$ & $43 \pm 1.9$ & 0.202 \\
\hline Colloid (ml) & $1081 \pm 53.3$ & $906 \pm 86.6$ & 0.089 \\
\hline Crystal (ml) & $1791 \pm 63.5$ & $1605 \pm 76.3$ & 0.065 \\
\hline Urine volume (ml) & $566 \pm 75$ & $535 \pm 55$ & 0.737 \\
\hline Blood loss (ml) & $150 \pm 20$ & $145 \pm 18$ & 0.853 \\
\hline Operation time (min) & $146.7 \pm 7.7$ & $141.9 \pm 8.6$ & 0.676 \\
\hline Anesthesia time (min) & $171.3 \pm 7.8$ & $170.4 \pm 9.2$ & 0.939 \\
\hline Pneumoperitoneum time (min) & $106.3 \pm 6$ & $103.2 \pm 7$ & 0.747 \\
\hline Time to extubation(min) & $16.83 \pm 4.97$ & $11.06 \pm 4.33$ & 0.001 \\
\hline Total time in PACU(min) & $34.63 \pm 5.42$ & $26.48 \pm 5.21$ & 0.001 \\
\hline Apnea immediately after extubation(n) & 2 & 1 & 1.000 \\
\hline Sp02 $<93 \%$ immediately after extubation & 4 & 5 & 1.000 \\
\hline Apnea within 30 min of extubation & 0 & 0 & \\
\hline SpO2 $<93 \%$ within 30 min of extubation & 0 & 0 & \\
\hline \multicolumn{4}{|l|}{ VAS } \\
\hline 1 hours post & $2.73 \pm 0.93$ & $1.67 \pm 0.94$ & 0.001 \\
\hline 4 hours post & $3.58 \pm 0.71$ & $2.73 \pm 0.60$ & 0.001 \\
\hline 8 hours post & $4.10 \pm 0.78$ & $3.88 \pm 0.88$ & 0.230 \\
\hline
\end{tabular}




\begin{tabular}{|llll|}
\hline & $\begin{array}{l}\text { D Group } \\
(\mathbf{n = 4 0 )}\end{array}$ & $\begin{array}{l}\text { M Group } \\
(\mathbf{n = 4 0 )}\end{array}$ & $P$ \\
\hline Total bolus times within 24h & $10.40 \pm 1.92$ & $8.28 \pm 1.72$ & 0.001 \\
\hline VAS = visual analogue scale & & & \\
\hline
\end{tabular}

The VAS of the M Group was significantly lower at $1(2.73 \pm 0.93$ vs $1.67 \pm 0.94, P<0.01)$ and $4(3.58 \pm 0.71$ vs $2.73 \pm 0.60, P<0.01)$ hours after the operation. Total bolus times within $24 \mathrm{~h}(10.40 \pm 1.92$ vs $8.28 \pm 1.72$, $P<0.01)$ were less in M Group. There was no significant difference in VAS between the two groups at 8 hours after operation. The time to extubation $(16.83 \pm 4.97 \mathrm{~min} v s 11.06 \pm 4.33 \mathrm{~min}, P<0.01)$ and the total time in PACU $(34.63 \pm 5.42$ min vs $26.48 \pm 5.21 \mathrm{~min}, P<0.01)$ was significantly less in M Group.

Adverse events, including apnoea and desaturation ( $\mathrm{SpO} 2<93 \%)$ immediately after extubation and within 30 min of extubation are summarised in Table 3. The differences were of no statistical significance. All events of desaturation were resolved using the jaw-thrust manoeuvre and supplementary oxygen.

There were no significant differences in systolic and diastolic blood pressure and heart rate between the two groups before the operation, during the induction of anesthesia, one or two hours into the operation, and after the operation (Fig. 3).

\section{Discussion}

Our study has shown that in laparoscopic colorectal surgery, moderate neuromuscular blockade combined with TAPB provides surgical space conditions similar to deep neuromuscular blockade.

In recent years, a growing number of anesthesiologists have applied deep neuromuscular blockade technology in laparoscopic surgery to provide surgeons with better surgical space conditions so as to improve abdominal organ perfusion and alleviate postoperative pain for laparoscopic surgery.[19-22] The results of a meta-analysis by Bruintjes et al. demonstrate that deep neuromuscular blockade improves surgical space conditions during laparoscopic surgery compared with moderate neuromuscular blockade. This study quantified surgical space conditions by measuring the distance from the sacral promontory to the umbilical skin. The results indicate that deep neuromuscular blockade increased the distance from the sacral promontory to the umbilical skin.[5] Author's study used this method to evaluate the surgical conditions during laparoscopic surgery. There was no significant difference between $\mathrm{M}$ Group and G Group in surgical space measurements.

A study on bariatric surgery published in PLOS One in 2016 included 100 obese patients. The researchers randomly divided all patients into moderate and deep neuromuscular blockade groups. Surgeons evaluated the surgical space conditions according to the operation evaluation scale. The results showed 
that in the deep neuromuscular blockade group, the surgeon's operation evaluation results were maintained five points, and the patients suffered from less pain in the post-anesthesia care unit $(P<$ 0.05), including less shoulder pain $(P<0.05)$.[16] Our results show that both moderate neuromuscular blockade combined with TAPB and deep neuromuscular blockade can provide a excellent surgical space conditions.

Furthermore, studies have exhibited reduced muscle injury after deep neuromuscular blockade during total hip replacement and that the levels of IL-6, CPK, and LDH were lower after the operation. $[23,24]$ Deep neuromuscular blockade can help to reduce muscle injury and reduce the stress of an operation and perioperative inflammation to a certain extent through improving surgical space conditions and making the operation easier to perform for the surgeon.[25-27] But it is not so easy to popularize deep neuromuscular blockade because of the prolonged recovery time and increased incidence of residual neuromuscular blockade after the operation.[11] Another possible reason can be that Sugammadex, the rocuronium specific antagonist, is quite expensive in China and out of the medical insurance list. Our study showed a prolonged extubation time and the PACU residence time in the D Group. Kopman et al. proposed that the antagonism of neostigmine was slow and incomplete when using deep neuromuscular blockade, leading to an increase in the incidence of postoperative residual neuromuscular blockade.[28]

Research showed that local anesthetics can enhance the effect of muscle relaxants caused by rocuronium.[15] Wang et al. found that the increased adult muscle-type nicotinic acetylcholine receptor inhibition produced when local anesthetics are combined with nondepolarizing muscle relaxants may contribute to the clinical enhancement of neuromuscular blockade by local anesthetics.[14] These studies may explain that moderate neuromuscular blockade combined with TAPB can achieve similar effect of deep muscle relaxation in our study.

The application of deep neuromuscular blockade in laparoscopic surgery is recognized as the optimal choice (keeping the TOF count at 0 and PTC $\geq 1$ ). 5 Compared with simple deep neuromuscular blockade, deep neuromuscular blockade combined with TAPB can provide good surgical space conditions and exert an appropriate analgesic effect,31 such that deep neuromuscular blockade combined with TAPB group was not included in this study. During our pilot study, three comparison groups were investigated, including deep neuromuscular blockade, moderate neuromuscular blockade combined with TAPB, and moderate neuromuscular blockade alone. Surgeons complained of a poor neuromuscular blockage effect for five patients in the moderate neuromuscular blockade group, so additional neuromuscular blockade was required to complete the operation. Consequently, this study eliminated the moderate neuromuscular blockade group.

In this study, the TAPB technique was guided by ultrasound, for which the accurate and safe operation has been mastered by most anesthesiologists. Similar to previous researchs,[29, 30] TAPB alleviated the pain within 4 hours after operation slightly in author's study.

There were several limitations to this study. First, the sample size of this study is relatively small, and it is likely underpowered to detect a difference in some adverse events, like apnea and desaturation. The 
major side effects of neuromuscular blockade focus on postoperative respiratory function, including postoperative hypoxemia, airway obstruction, and muscle weakness, resulting in an increased risk of postoperative respiratory complications.[12] Second, we did not compare the lengths of the hospital stay and ICU stay and we did not collect long-term follow-up data from the patients.

\section{Conclusion}

In laparoscopic colorectal cancer surgery, moderate neuromuscular blockade combined with TAPB can provide surgical space conditions similar to those of deep neuromuscular blockade, and the same time, reduces the use of muscle relaxants, relieves postoperative pain within 4 hours after operation, shorten the time to extubation and stay in PACU.

\section{Abbreviations}

TAPB: transverse abdominal plane block; TOF: train-of-four; PTC: post-tetanic count; VAS: visual analogue scale; MAC: minimum alveolar concentration; ASA: American Society of Anesthesiologists; BMI: body mass index; TCl: target control infusion; MAP: mean arterial pressure; PCA: patient controlled analgesia

\section{Declarations}

\section{Acknowledgements}

We would like to thank MogoEdit (en.mogoedit.com) for English language editing.

\section{Authors' contributions}

RD, FK, ZJS, CW helped in study design. FK and ZJS helped in patient recruitment and data collection. CW helped in statistical analysis. FK wrote the first draft of the manuscript. RD, ZJS and LZ help in critical revision. All authors have read and approved the manuscript.

\section{Funding}

Supported by Foundation of Science and Technology Commission of Shanghai Municipality(No. 20035800600), Foundation of Shanghai Municipal Health Commission(No. 20184Y0088), Foundation of Shanghai Municipal Health Commission(No. 202040017), Foundation of Ruijin Hospital(No. 2019ZY15)

\section{Availability of data and materials}

The datasets used and/or analysed during the current study are available from the corresponding author on reasonable request.

\section{Ethics approval and consent to participate}


This trail was approved by the ethics committee of the Ruijin Hospital affiliated to the Shanghai Jiaotong University School of Medicine (No. 2020-007-1) and written informed consent was obtained from each participant.

\section{Consent for publication}

Not applicable.

\section{Competing interests}

The authors declare that they have no competing interests.

\section{Author details}

${ }^{1}$ Department of Anesthesiology, Ruijin Hospital affiliated to Shanghai Jiaotong University School of Medicine, Shanghai 200025, China

${ }^{2}$ Department of Health Statistics $₫$ Naval Medical University, Shanghai 200433, China

\section{References}

1. King $M$, Sujirattanawimol N, Danielson DR, Hall BA, Schroeder DR, Warner DO: Requirements for muscle relaxants during radical retropubic prostatectomy. Anesthesiology 2000, 93:1392-1397.

2. Bai Y, Ren H, Luo A, Huang Y, Ye T, Guo X: Effects of residual paralysis after a single intubating dose of rocuronium on postoperative pulmonary function of patients undergoing laparoscopic gynecological surgeries. Acta academiae medicinae sinicae 2010, 32:103-107.

3. Cho YJ, Paik H, Jeong SY, Park JW, Jo WY, Jeon Y, Lee KH, Seo JH: Lower intra-abdominal pressure has no cardiopulmonary benefits during laparoscopic colorectal surgery: a double-blind, randomized controlled trial. Surg Endosc 2018, 32:4533-4542.

4. Diaz-Cambronero O, Flor Lorente B, Mazzinari G, Vila Montañes M, García Gregorio N, Robles Hernandez D, Olmedilla Arnal LE, Argente Navarro MP, Schultz MJ, Errando CL: A multifaceted individualized pneumoperitoneum strategy for laparoscopic colorectal surgery: a multicenter observational feasibility study. Surg Endosc 2019, 33:252-260.

5. Bruintjes MH, van Helden EV, Braat AE, Dahan A, Scheffer GJ, van Laarhoven CJ, Warlé MC. Deep neuromuscular block to optimize surgical space conditions during laparoscopic surgery: a systematic review and meta-analysis. Br J Anaesth 2017, 118:834-842.

6. Jacob R, Joseph HW, Manfred B, Mulier Jan P, Niels R, Tiffanyl W, Li Michael K, Peter G, Assaid Christopher A, Hein F, Armin S. Deep Neuromuscular Blockade Improves Laparoscopic Surgical Conditions: A Randomized, Controlled Study. Adv Ther 2017, 34:925-936.

7. Özdemir-van Brunschot D M D, Braat A E, van der Jagt M F P, Scheffer $G J$, Martini $C H$, Langenhuijsen J F, Dam R E, Huurman V A, Lam D, d'Ancona F C, Dahan A, Warlé M C. Deep 
neuromuscular blockade improves surgical conditions during low-pressure pneumoperitoneum laparoscopic donor nephrectomy. Surg Endosc, 32(1), 2018, 32:245-251.

8. Bruintjes Moira H D, Piet K, Martini Chris H, Poyck Paul P, d'Ancona Frank C H, Huurman Volkert A L, van der Jagt Michel, Langenhuijsen Johan F, Nijboer Willemijn N, van Laarhoven Cornelis J H M, Albert D, Warlé Michiel C, RELAX cg: Efficacy of profound versus moderate neuromuscular blockade in enhancing postoperative recovery after laparoscopic donor nephrectomy: A randomised controlled trial. Eur J Anaesthesiol 2019, 36:494-501.

9. Dubois Philippe E, Laurie P, Jacques J, Maria-Laura M, Maximilien G, Donnez O: Deep neuromuscular block improves surgical conditions during laparoscopic hysterectomy: a randomised controlled trial. Eur J Anaesthesiol 2014, 31:430-436.

10. Marjolijn L, Ulas BL, Apers Jan A, Erwin B, Verbrugge Serge J C, Dunkelgrun M: Low-pressure pneumoperitoneum with deep neuromuscular blockade in metabolic surgery to reduce postoperative pain: a randomized pilot trial. Surg Endosc 2020:Published online.

11. Fuchs-Buder T, Nemes R, Schmartz D. Residual neuromuscular blockade: management and impact on postoperative pulmonary outcome. Curr Opin Anaesthesiol 2016, 29:662-667.

12. Kirmeier E, Eriksson LI, Lewald H, Jonsson Fagerlund M, Hoeft A, Hollmann M, Meistelman C, Hunter JM, UIm K, Blobner M: Post-anaesthesia pulmonary complications after use of muscle relaxants (POPULAR): a multicentre, prospective observational study. Lancet Respir Med 2019, 7: 129-140.

13. McLean Duncan J, Daniel D, Farhan Hassan N, Ladha Karim S, Tobias K, Matthias E: Dose-dependent Association between Intermediate-acting Neuromuscular-blocking Agents and Postoperative Respiratory Complications. Anesthesiology 2015, 122:1201-1213.

14. Wang H, Zhang Y, Li ST: The effect of local anesthetics on the inhibition of adult muscle-type nicotinic acetylcholine receptors by nondepolarizing muscle relaxants. Eur J Pharmacol 2010, 630:29-33.

15. Carvalho VH, Braga Ade F, Braga FS, Loyola YC, de Araujo DR, Mantovani M: The influence of lidocaine and racemic bupivacaine on neuromuscular blockade produced by rocuronium. A study in rat phrenic nerve-diaphragm preparation. Acta Cir Bras 2009, 24:211-215.

16. Torensma B, Martini CH, Boon M, Olofsen E, In 't Veld B, Liem RS, Knook MT, Swank DJ, Dahan A: Deep Neuromuscular Block Improves Surgical Conditions during Bariatric Surgery and Reduces Postoperative Pain: A Randomized Double Blind Controlled Trial. PLoS One 2016, 11:e0167907.

17. Martini CH, Boon M, Bevers RF, Aarts LP, Dahan A: Evaluation of surgical conditions during laparoscopic surgery in patients with moderate vs deep neuromuscular block. Br J Anaesth 2014, 112:498-505.

18. Naguib M, Brull SJ, Kopman AF, Hunter JM, Fülesdi B, Arkes HR, Elstein A, Todd MM, Johnson KB: Consensus Statement on Perioperative Use of Neuromuscular Monitoring. Anesth Analg 2018, 127:71-80.

19. Loh PS, Yeong CH, Masohood NS, Sulaiman N, Zaki RA, Fabell K, Abdullah B: Comparison of deep and moderate neuromuscular blockade in microwave ablation of liver tumours: a randomized- 
controlled clinical trial. Sci Rep 2021, 11:2299.

20. Zhu SJ, Zhang XL, Xie Q, Zhou YF, Wang KR: Comparison of the effects of deep and moderate neuromuscular block on respiratory system compliance and surgical space conditions during robotassisted laparoscopic radical prostatectomy: a randomized clinical study. J Zhejiang Univ Sci B 2020, 21:637-645.

21. Zhang XF, Li DY, Wu JX, Jiang QL, Zhu HW, Xu MY: Comparison of deep or moderate neuromuscular blockade for thoracoscopic lobectomy: a randomized controlled trial. BMC Anesthesiol 2018, 18:195.

22. Staehr-Rye AK, Rasmussen LS, Rosenberg J, Juul P, Lindekaer AL, Riber C, Gätke MR: Surgical space conditions during low-pressure laparoscopic cholecystectomy with deep versus moderate neuromuscular blockade: a randomized clinical study. Anesth Analg 2014, 119:1084-1092.

23. Oh CS, Lim HY, Jeon HJ, Kim TH, Park HJ, Piao L, Kim SH: Effect of deep neuromuscular blockade on serum cytokines and postoperative delirium in elderly patients undergoing total hip replacement: $A$ prospective single-blind randomised controlled trial. Eur J Anaesthesiol 2021, 38:S58-58S66.

24. Eun KJ, Kee MS, Eunji H, Dongchul L, Yeop KJ, Kwak HJ: Effects of deep neuromuscular block with low-pressure pneumoperitoneum on respiratory mechanics and biotrauma in a steep Trendelenburg position. Sci Rep 2021, 11:1935.

25. Mulier Jan P, Dillemans B: Anaesthetic Factors Affecting Outcome After Bariatric Surgery, a Retrospective Levelled Regression Analysis. Obes Surg 2019, 29:1841-1850.

26. Kyeong OS, Woo-Keun K, Sangwoo P, Gi JS, Han KJ, Youn-Kwan P, Young LS, Lim BG: Comparison of Operating Conditions, Postoperative Pain and Recovery, and Overall Satisfaction of Surgeons with Deep vs. No Neuromuscular Blockade for Spinal Surgery under General Anesthesia: A Prospective Randomized Controlled Trial. J Clin Med 2019, 8:498.

27. Koo C H, Chung S H, Kim B G, Min B H, Lee SC, Oh A Y, Jeon Y T, Ryu JH: Comparison between the effects of deep and moderate neuromuscular blockade during transurethral resection of bladder tumor on endoscopic surgical condition and recovery profile: a prospective, randomized, and controlled trial. World J Urol 2019, 37:359-365.

28. Kopman AF, Naguib M: Laparoscopic surgery and muscle relaxants: is deep block helpful. Anesth Analg 2015, 120:51-58.

29. Børglum J, Gögenür l, Bendtsen TF: Abdominal wall blocks in adults. Curr Opin Anaesthesiol 2016, 29:638-643.

30. Mirra A, von Rotz A, Schmidhalter M, Moser L, Casoni D, Spadavecchia C. Ultrasound-guided lateral and subcostal transversus abdominis plane block in calves: a cadaveric study. Vet Anaesth Analg 2018, 45:384-391.

\section{Figures}




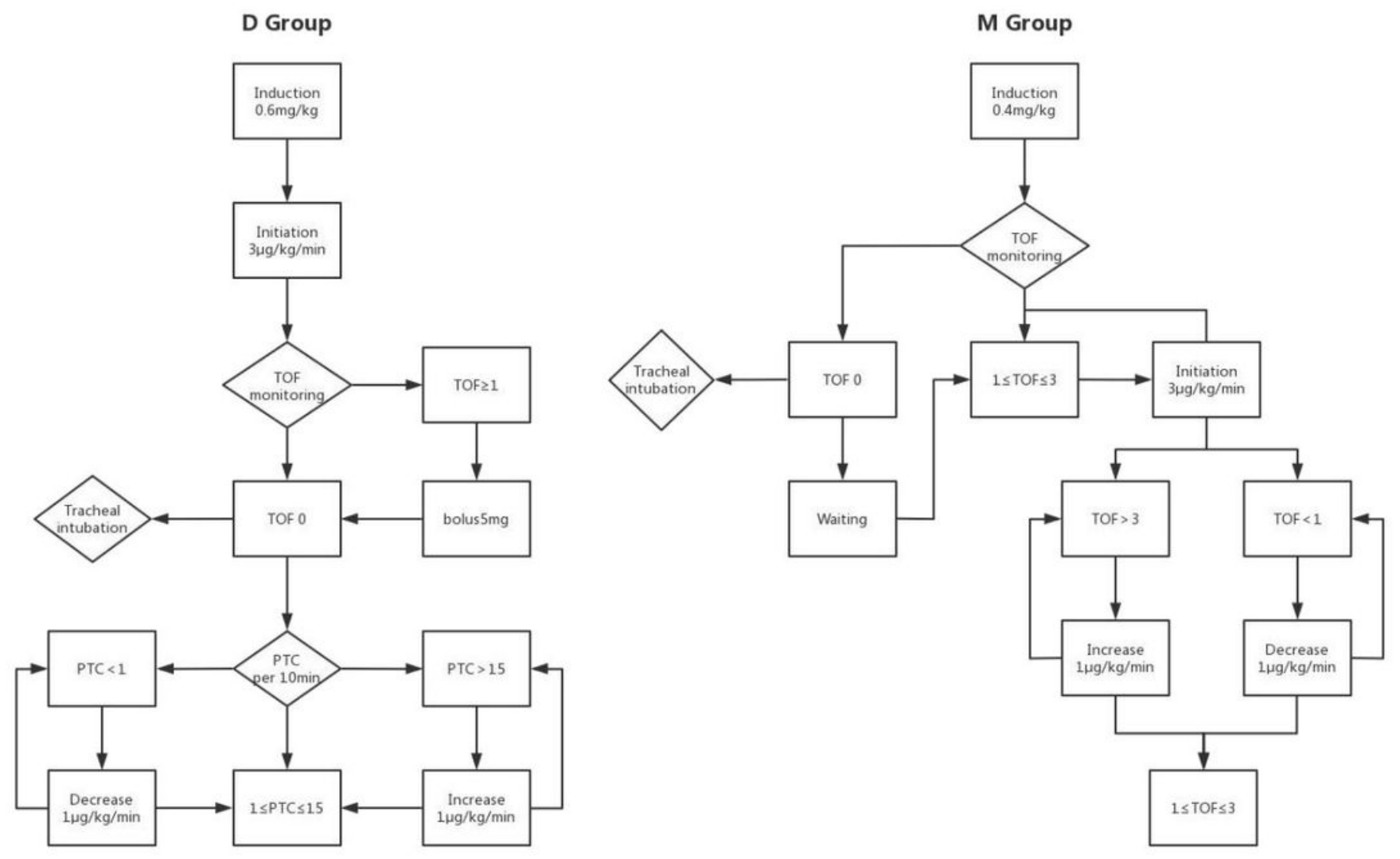

Figure 1

Flow chart of rocuronium management during surgery. TOF = train-of-four; PTC = post-tetanic count. 


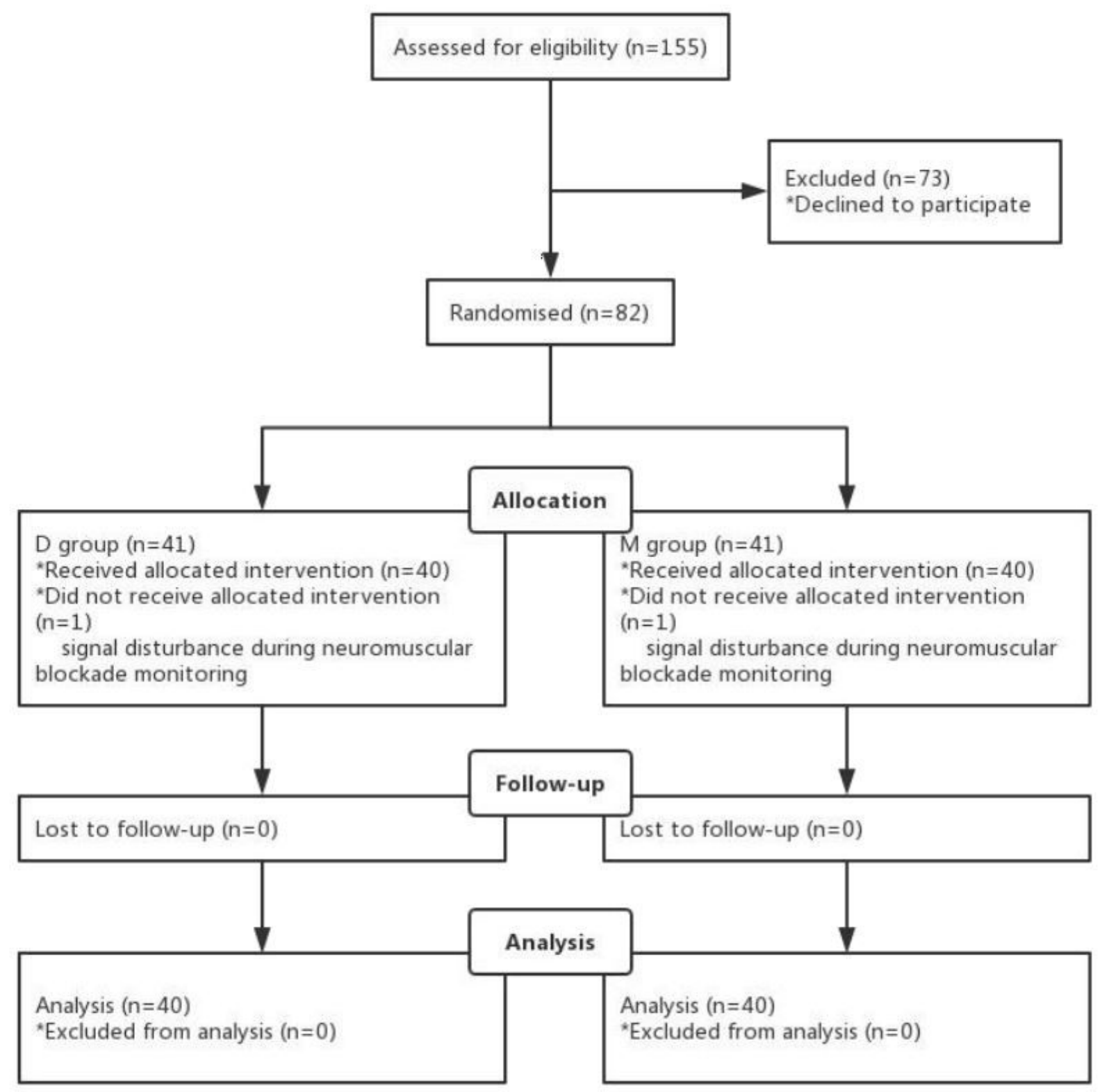

Figure 2

Consolidated Standards of Reporting Trials flow diagram 


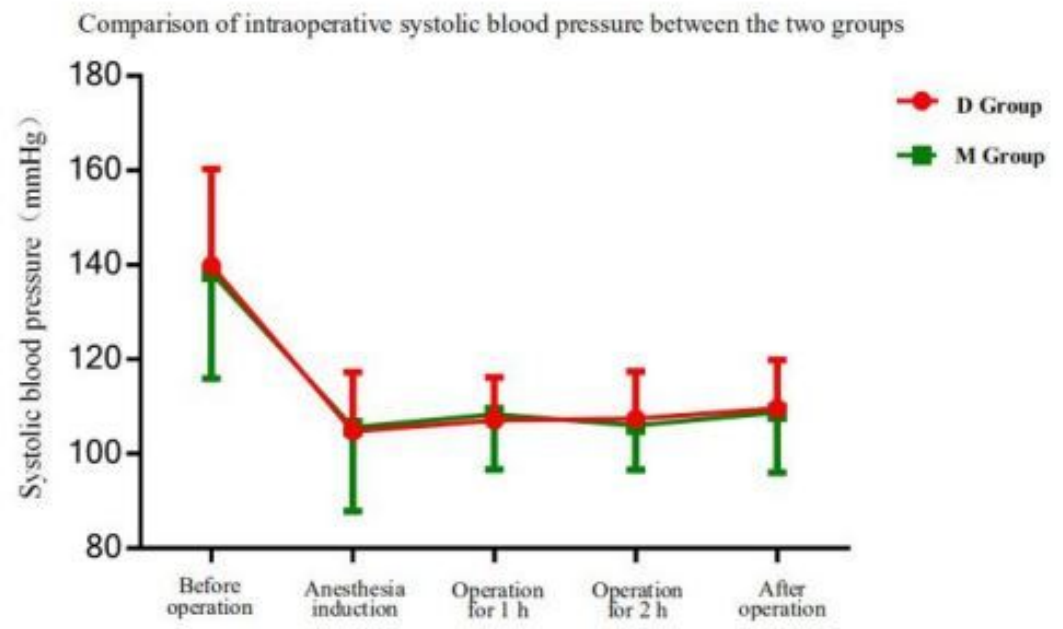

Comparison of intraoperative diastolic blood pressure between the two groups
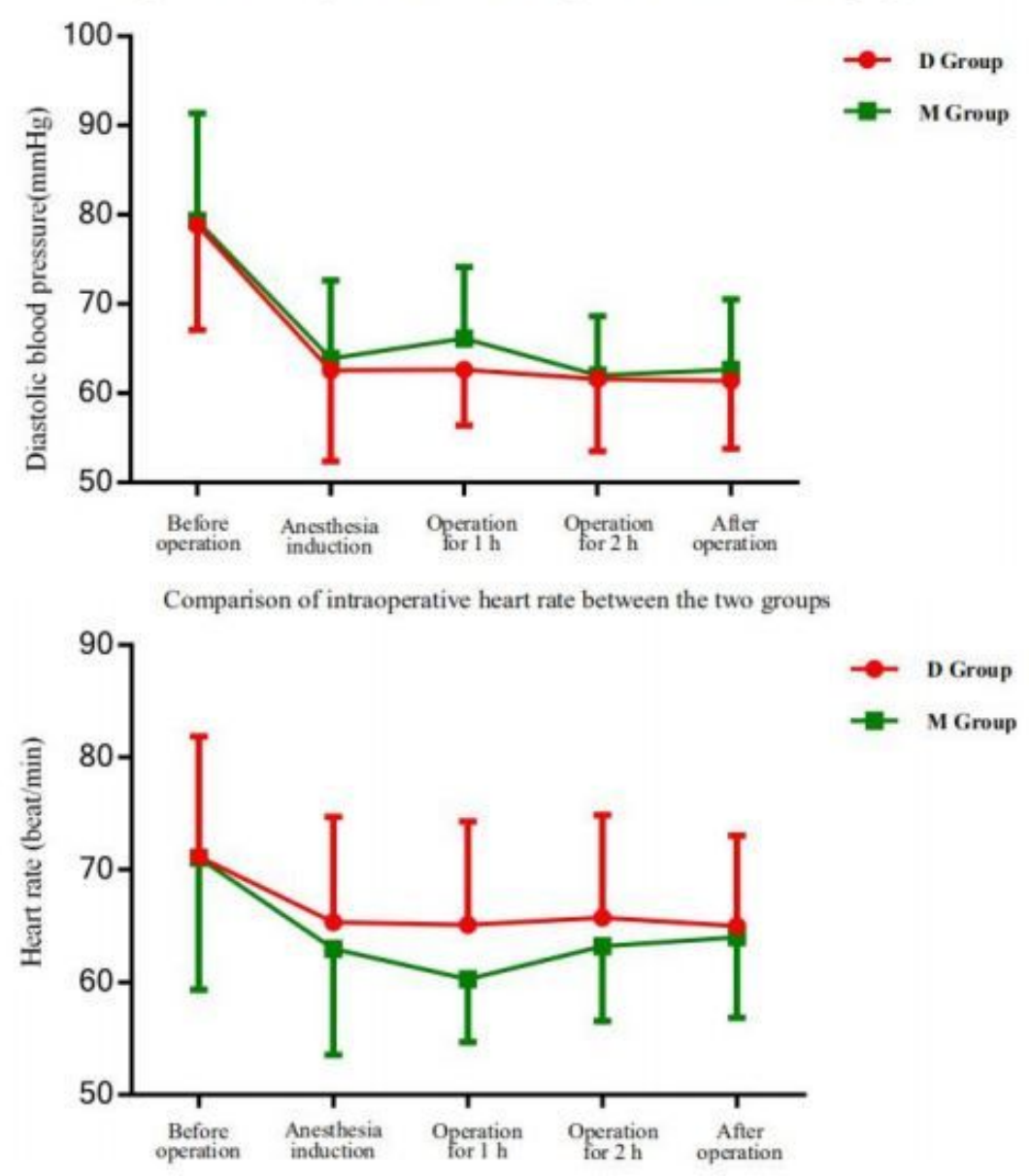

\section{Figure 3}

Comparison of intraoperative systolic blood pressure, diastolic blood pressure and heart rate between the two groups 